\title{
Comparison of hCG Versus Gonadotropin-Releasing Hormone Agonist to Induce Oocyte Maturation in Assisted Reproductive Technology Cycles: A Retrospective Cohort Study
}

Gulay BEYDILLI NACAK ${ }^{1}$, Elif TOZKIR ${ }^{1}$, Enis OZKAYA ${ }^{1}$, Ebru COGENDEZ ${ }^{1}$, Fatih KAYA ${ }^{1}$

Istabul, Turkey

\section{ABSTRACT}

OBJECTIVE: To compare some cycle characteristics and outcomes using a protocol consisting of a $\mathrm{GnRH}$ agonist trigger or hCG trigger after cotreatment with $\mathrm{GnRH}$ antagonist.

STUDY DESIGN: Thirty-three patients under 35 years of age with polycystic ovarian syndrome, polycystic ovarian morphology, or previous high response who underwent ovulation trigger by $\mathrm{GnRH}$ agonist trigger and 132 patients under 35 years of age with the polycystic ovarian syndrome, polycystic ovarian morphology, or previous high response who underwent ovulation trigger by hCG for IVF treatment. Patients were non-randomly assigned to an ovarian stimulation protocol consisting of either $\mathrm{GnRH}$ agonist trigger after cotreatment with $\mathrm{GnRH}$ antagonist (study group) or hCG trigger after antagonist protocol (control group).

RESULTS: The positive pregnancy test was obtained in 70 women in the control group whereas in 13 cases in the study group $(p=0.161)$. No case in the study group needed hospitalization whereas there were 15 cases in the control group who were required to be hospitalized due to ovarian hyperstimulation related symptoms $(p=0.04)$.

CONCLUSIONS: The use of a protocol consisting of a $\mathrm{GnRH}$ agonist trigger after $\mathrm{GnRH}$ antagonist cotreatment and freeze-all strategy reduces the risk of ovarian hyperstimulation syndrome in high-risk patients undergoing IVF without affecting pregnancy rates.

Keywords: GnRH agonist, GnRH antagonist, In vitro fertilization, Ovarian hyperstimulation syndrome, Polycystic ovary morphology, Polycystic ovary syndrome

Gynecol Obstet Reprod Med 2021;27(2):143-149

\section{Introduction}

Ovarian hyperstimulation syndrome (OHSS) is an iatrogenic complication of controlled ovarian hyperstimulation

\footnotetext{
${ }^{I}$ Department of Obstetrics and Gynecology, Health Sciences University Zeynep Kamil Women and Children's Diseases Training and Research Hospital, Istanbul, Turkey

Address of Correspondence: Enis Ozkaya

Department of Obstetrics and Gynecology,

Health Sciences University Zeynep Kamil

Women and Children's Diseases Training

and Research Hospital, $34668 \ddot{\text { Usküdar, }}$

Istanbul, Turkey

enozkaya1979@gmail.com
}

Submitted for Publication:12.10.2019 Revised for Publication:16.12.2019 Accepted for Publication: 28.12.2019 Online Published: 02.08.2021

ORCID IDs of the authors: ET: 0000-0001-6996-4615

GBN: 0000-0001-5452-4561

EO: 0000-0001-6580-1237 EC: 0000-0001-7062-3076

FK: 0000-0001-7001-6954

\begin{tabular}{c|c}
\hline Quick Response Code: & Access this article online \\
\cline { 2 - 2 } & Website: www.gorm.com.tr \\
& e- mail: info@gorm.com.tr \\
\hline
\end{tabular}

How to cite this article: Beydilli Nacak G. Tozkir E. Ozkaya E. Cogendez E. Kaya F. Comparison of hCG Versus Gonadotropin-Releasing Hormone Agonist to Induce Oocyte Maturation in Assisted Reproductive Technology Cycles:A Retrospective Cohort Study. Gynecol Obstet Reprod Med. 2021;27(2):143-149
$(\mathrm{COH})$, which may result in severe morbidity and rarely mortality (1). Although there has been significant progress over the years in assisted reproductive technology, there is currently no effective strategy to address this disorder (2). One of the best strategies for the prevention of OHSS is the identification of high-risk patients and the use of an appropriate ovarian stimulation protocol. High-risk patients include young women with polycystic ovary syndrome (PCOS) $(3,4)$ and patients with a history of previous high response to gonadotropins. It has been suggested that the administration of a GnRH agent to induce the final oocyte maturation may lead to a reduction in the risk of OHSS $(5,6)$. This is due to the shorter half-life of endogenous LH fluctuation and subsequent suppression of the pituitary, leading to early luteolysis and decreased luteal phase steroidal concentration (7). On the other hand, there is insufficient data in the literature in terms of cycle characteristics and success following the agonist trigger.

The aim of this study was to compare some cycle characteristics and outcome of cycles using a protocol consisting of GnRH agonist trigger after cotreatment with $\mathrm{GnRH}$ antagonist or hCG trigger in antagonist protocols

\footnotetext{
(cc) BY Copyright ${ }^{\circ}$ 2021. Beydilli Nacak et al. This article is distributed under a Creative Commons Attribution 4.0 International License.
} 


\section{Material and Method}

This was a retrospective cohort study involving patients undergoing In vitro fertilization (IVF) treatment at the ART (assisted reproductive technology) center of Health Sciences University Zeynep Kamil Women and Children's Health Training and Research Hospital. The subjects were recruited for a period of 48 months between January 2015 and January 2019. This study was conducted in accordance with the Declaration of Helsinki. Approval for this study was obtained from the Institutional Review Board (2016/74).

Participants: Patients were enrolled in the study if they fulfilled the following criteria: age 23-35 years at the time of screening, normal early follicular phase serum FSH concentration $(<10.0 \mathrm{IU} / \mathrm{L})$, and undergoing their first cycle of IVF with either PCOS or PCOM (polycystic ovary morphology) or undergoing a subsequent cycle with a history of high response in a previous IVF cycle. Polycystic ovary syndrome was defined according to the Rotterdam consensus guidelines (8), and the evaluation of PCOM was based on the international consensus definitions (9). Previous high responders were patients who either developed OHSS underwent coasting, or had a cycle canceled for risk of OHSS in a previous cycle.

Consecutive thirty-three patients under 35 years of age with polycystic ovarian syndrome, polycystic ovarian morphology, or previous high response undergoing IVF who underwent ovulation trigger by GnRH agonist and again consecutive 132 patients under 35 years of age with polycystic ovarian syndrome, polycystic ovarian morphology, or previous high response undergoing IVF who underwent ovulation trigger by hCG were included in this study.

Treatment Protocol: For all participants, a gonadotropinsecreting hormone antagonist protocol was used for IVF/ICSI. On the second day of the menstrual cycle, the daily folliclestimulating hormone (rFSH; Gonal-F, Merck-Serono, Geneva, Switzerland) was initiated. The dose used ranged from $150 \mathrm{IU}$ to $300 \mathrm{IU}$ and was determined by the baseline clinical characteristics of each patient. Mean follicular growth was monitored by two-dimensional transvaginal sonography every 2-3 days. The daily dose of rFSH was adjusted from day 5 of stimulation according to the ovarian response. Antagonist (Cetrorelix, Merck-Sereno, Geneva, Switzerland) was administered at a dose of $0.25 \mathrm{mg}$ /day when the follicular size reached $12 \mathrm{~mm}$. When the follicular size reached $18 \mathrm{~mm}, 250$ microg recombinant human chorionic gonadotropins (hCG; Ovitrelle, Merck-Sereno, Geneva, Switzerland) was administered subcutaneously and follicular puncture was performed 34-36 hours later. Then, 8\% vaginal progesterone gel (Crinone gel 8\%; Merck-Sereno, Geneva, Switzerland) was applied twice daily. ICSI was performed for each oocyte obtained by follicular puncture. According to the developmental characteristics of the embryo, the elective transfer of an embryo was performed at the cleavage (day 3 ) or blastocyst (day
5) stage. Serum levels of the hCG ( $\beta$-hCG) G-subunit were measured after 2 weeks. The patient was considered to have successful implantation if it was equal to or greater than normal levels (5 IU/L) during pregnancy. Patients in the study group, final oocyte maturation was triggered by administering $0.2 \mathrm{mg}$ triptorelin (Decapeptyl; Ferring, Turkey) approximately 12 hours after the last dose of ganirelix.

Transvaginal ultrasound-directed oocyte retrieval was performed 35 hours after $\mathrm{GnRH}$ or hCG administration. Embryo transfer was performed 72 to 76 hours after oocyte retrieval. Supernumerary multicellular embryos of good quality or blastocysts were then cryopreserved on days 3 and/or 5 respectively in the group of women who underwent agonist trigger. The embryos were graded according to the criteria described by Cummins et al. (10). Timing and endometrial preparations for Frozen embryo transfers were determined according to the article by Mackens et al (11). Estradiol hemihydrate $2 \mathrm{mg} 3$ times/day was administered orally under transvaginal monitorization with 2-3 days intervals until the endometrial thickness reached 9-14 $\mathrm{mm}$. Then intravaginal progesterone gel was administered. The duration of luteal phase supplementation was determined according to the day of the frozen embryo (day of frozen embryo+1).

Outcome Variables: The primary efficacy endpoint was the incidence of OHSS and the secondary endpoint pregnancy rate. Additional points of interest were the number of oocytes taken, the ratio of mature oocytes obtained, the rate of fertilization. OHSS was diagnosed by Golan et al. (12). All patients were brought back after 7 days of oocyte uptake (mid-luteal phase) and their symptoms, findings, sonographic and laboratory evidence were evaluated.

Statistical analyses were performed using Statistical Package for Social Sciences (Publication 17.0; SPSS, Chicago, IL). When appropriate, chi-square or Fisher exact tests were used for categorical variables. Independent samples t-test was used for continuous variables with normal distribution and the Mann-Whitney U test was used for data with no normal distribution. All $\mathrm{p}$ values quoted are bilateral and values below .05 are taken to indicate statistical significance.

\section{Results}

Comparison of groups in terms of some demographic characteristics was summarized in table I which showed similar age, BMI and duration of infertility ( $p>0.05$, Table I). The primary infertility rates were similar between the two groups $(81.8 \%$ vs. $78.8 \%, p>0.05)$. The rates of patients with PCOS were also similar between the two groups $(50.8 \%$ vs. $51.5 \%$, $p>0.05$ ). Again, the rates of patients with previous IVF trials were similar between groups with and without agonist trigger (32.6\% vs. $33.4 \%, p>0.05)$. Baseline ovarian reserve tests were similar between groups ( $p>0.05$, Table II). The coasting procedure was required in $4(12.1 \%)$ cases with agonist trig- 
ger, on the other hand, $50(37.9 \%)$ cases in the group with hCG trigger $(p=0.005)$. Oocyte fertilization rates did not differ between the two groups $(94.7 \%$ vs. $93.9 \%, p>0.05)$. Different embryo grade rates were comparable between the two groups ( $p>0.05$, Table III). Among 33 women who un- derwent agonist trigger, freeze all strategy was indicated for 21 cases, whereas among 132 women in the control group freeze all strategy was preferred in 10 cases $(p<0.001)$. All of the parameters representing the cycle characteristics were similar between groups $(p>0.05$, Table IV). Positive preg-

Table I: Comparison of groups in terms of some demographic characteristics

\begin{tabular}{llllll}
\hline & Trigger & $\mathrm{n}$ & Mean & Std. deviation & $p$ value \\
\hline Age $($ Years) & Agonist & 33 & 29.7 & 4.01 & $0.6(\mathrm{MWU})$ \\
& hCG & 132 & 30.1 & 4.4 & 5.2 \\
BMI $\left(\mathrm{kg} / \mathrm{m}^{2}\right)$ & Agonist & 17 & 24.7 & 3.1 & 0.09 \\
Duration of infertility (months) & hCG & 51 & 23.1 & 35.5 & $0.3(\mathrm{MWU})$ \\
& Agonist & 33 & 44.2 & 40.8 & 0.5 \\
\hline
\end{tabular}

BMI: Body mass index, MWU: Mann Whitney-U

Table II: Baseline ovarian reserve tests were similar between groups

\begin{tabular}{llllll}
\hline & Trigger & $\mathrm{n}$ & Mean & Std. deviation & $p$ value \\
\hline FSH $(\mathrm{mlU} / \mathrm{mL})$ & hCG & 132 & 5.150 & 1.3310 & 0.5 \\
Estradiol $(\mathrm{pg} / \mathrm{mL})$ & Agonist & 33 & 5.034 & 1.4141 & 15.726 \\
AFC & hCG & 132 & 40.91 & 17.996 & $0.2(\mathrm{MWU})$ \\
& Agonist & 33 & 45.67 & 6.014 & $0.5(\mathrm{MWU})$ \\
\hline
\end{tabular}

FSH: Follicle stimulating hormone, AFC: Antral follicle count, MWU: Mann Whitney-U

Table III: Different embryo grade rates were comparable between the two groups

\begin{tabular}{|c|c|c|c|c|c|c|}
\hline & & Embryc & & & & $p$ value \\
\hline & & 1 & 2 & 3 & 4 & \\
\hline \multirow[t]{4}{*}{ Trigger } & hCG & 81 & 20 & 2 & 21 & \\
\hline & & $65.3 \%$ & $16.1 \%$ & $1.6 \%$ & $16.9 \%$ & \\
\hline & Agonist & 19 & 4 & 2 & 6 & 0.5 \\
\hline & & $61.3 \%$ & $12.9 \%$ & $6.5 \%$ & $19.4 \%$ & \\
\hline \multirow[t]{2}{*}{ Total } & & 100 & 24 & 4 & 27 & \\
\hline & & $64.5 \%$ & $15.5 \%$ & $2.6 \%$ & $17.4 \%$ & \\
\hline
\end{tabular}

hCG: Human chorionic gonadotropin

Table IV: All of the parameters of representing the cycle characteristics were similar between groups

\begin{tabular}{|c|c|c|c|c|c|}
\hline & Trigger & $\mathrm{n}$ & Mean & Std. deviation & $p$ value (MWU) \\
\hline \multirow[t]{2}{*}{ Starting Gonadotropin Dose (U) } & hCG & 132 & 208.7 & 58.6 & \\
\hline & Agonist & 33 & 212.8 & 58.6 & 0.7 \\
\hline \multirow[t]{2}{*}{ Total Gonadotropin Dose (U) } & hCG & 132 & 1856.4 & 909.8 & \\
\hline & Agonist & 33 & 1931.4 & 727.5 & 0.2 \\
\hline \multirow[t]{2}{*}{ Day 5 Estradiol (pg/mL) } & hCG & 132 & 800.9 & 502.6 & \\
\hline & Agonist & 33 & 825.3 & 566.9 & 0.9 \\
\hline \multirow[t]{2}{*}{ Duration of Induction } & hCG & 132 & 8.8 & 1.6 & \\
\hline & Agonist & 33 & 9.1 & 2.05 & 0.3 \\
\hline \multirow[t]{2}{*}{ Peak Estradiol (pg/mL) } & hCG & 132 & 4272.09 & 1964.8 & \\
\hline & Agonist & 33 & 4060.5 & 1161.9 & 0.5 \\
\hline \multirow[t]{2}{*}{ \# of total oocytes } & $\mathrm{hCG}$ & 132 & 12.2 & 5.7 & \\
\hline & Agonist & 33 & 13.8 & 6.08 & 0.2 \\
\hline \multirow[t]{2}{*}{ \# of mature oocytes } & hCG & 132 & 9.6 & 4.9 & \\
\hline & Agonist & 33 & 11.2 & 5.2 & 0.1 \\
\hline \multirow[t]{2}{*}{ Fertilization rate $(\%)$} & hCG & 131 & 90 & 20 & \\
\hline & Agonist & 33 & 94 & 24.2 & 0.9 \\
\hline
\end{tabular}

\#: number, MWU: Mann Whitney-U 
nancy tests were obtained in 70 women in the control group whereas in 13 cases in the study group $(p=0.161)$. There was a single case with mild OHSS in the study group whereas, in the control group, 19 OHSS cases were observed ( $p=0.07$ ). No case in the study group needed hospitalization whereas 15 cases in the control group required hospitalization ( $p=0.04)$.

\section{Discussion}

In this study, we aimed to compare some characteristics and outcomes of cycles using a protocol consisting of $\mathrm{GnRH}$ agonist trigger after cotreatment with GnRH antagonist or $\mathrm{hCG}$ trigger in antagonist protocols. Our comparison revealed that the use of a protocol consisting of GnRH agonist trigger after GnRH antagonist cotreatment and freeze-all strategy reduces the risk of OHSS in high-risk patients undergoing IVF without affecting pregnancy rates.

To date, various strategies have been proposed for the prevention of OHSS in high-risk patients, but none have been shown to eliminate the risk of OHSS (2). Previous studies have shown that the induction of oocyte maturation with the GnRH agonist after GnRH antagonist co-treatment can potentially prevent the development of OHSS $(5,6,13)$. On the other hand, there is insufficient data in the literature in terms of cycle characteristics and success following agonist trigger. The OHSS inhibition mechanism after the GnRH agonist trigger was not fully investigated. Administration of a single dose of the $\mathrm{GnRH}$ agonist causes an endogenous rise of both FSH and LH, resulting in the induction of the final stages of oocyte maturation, after bedtime with the GnRH antagonist $(14,15)$. The shorter half-life of the internal LH fluctuation and subsequent suppression of the pituitary and withdrawal of LH support for corpora lutea may lead to early luteolysis (7). The GnRH agonist has been advocated since 1989 to trigger oocyte maturation and ovulation (16). However, it is not widely accepted and widely used for various reasons. First, GnRH fell in favor in the 1990s due to the widespread use of agonist desensitization protocols; Second, one of the major disadvantages of the GnRH agonist trigger is the weaker induction of luteal phase and consequently impaired steroid circulation $(14,15)$. In fact, recent publications showed that the GnRH agonist trigger may result in impaired implantation and ongoing pregnancy rates in normal responders (17-19). In a previous study, two groups of patients who were assigned to receive either GnRHa trigger $(\mathrm{n}=98$ cycles $)$ or hCG trigger $(\mathrm{n}=99$ cycles $)$ for ovulation trigger were compared for intrauterine insemination (IUI) cycle outcome. No difference was found in ovulation rates in either group receiving $\mathrm{GnRHa}$ or hCG trigger for ovulation. Biochemical and clinical pregnancy rates were higher in group with hCG trigger $(28.3 \%$ and $23.2 \%)$ versus GnRHa $(14.3 \%$ and $13.3 \%)(p=0.023$, and $p=0.09$, respectively). However, this study showed that after adjusting for body mass index and infertility duration, there was no difference in clinical pregnancy rates between the two groups $(p=0.163$ ). The authors of this study concluded that the use of the GnRHa to trigger ovulation in patients undergoing ovulation induction may be considered in patients treated with IUI (20). In our study population cases who underwent the GnRHa trigger was required to be directed to the "Freeze-all" strategy. The "Freeze-all" strategy was questioned in the literature. The "Freeze-all" policy has been proposed for the general IVF population; (21) there are important concerns that need to be taken into consideration although the initial studies and a meta-analysis supported the use of "Freeze-all" policy for the general IVF population $(22,23)$, recent evidence from larger randomized clinical trials in more specific subpopulations does not support the general use of "Freeze-all" policy (24-26) moreover, some authors reported higher early pregnancy loss rates related to frozenthawed cycles, (27) as well as a higher incidence of adverse obstetrical and perinatal outcomes, (28) which might be induced by either the endometrial priming protocol prior to embryo transfer or the cryo-technique itself. In contrast, in patients with OHSS risk, the benefits from the "Freeze-all" policy may already outweigh all the reported risks (29). Hence, the clinician has the possibility to counterbalance benefits and risks in the OHSS risk patient by using either a fresh cycle with GnRHa trigger and modified luteal phase support or turning directly to "Freeze-all" policy. Another possible drawback of the GnRH trigger was suggested to be increased risk for empty follicle syndrome. Some studies have been published indicating this issue for example in the previous study, clinicians aimed to investigate the incidence of empty follicle syndrome (EFS) occurring in gonadotropin-releasing hormone agonist-induced IVF cycles in women with the polycystic ovarian syndrome and GnRH antagonist protocol. Following an ovulation trigger by GnRHa in the IVF cycle of 279 PCOS patients; the incidence of EFS following GnRHa was reported to be $3.3 \%$. Among these cases $88.8 \%$ (8/9) of EFSs had false empty follicle syndrome; Six of 9 cases were given a rescue trigger and a very high level of embryo transfer was reported in the FET cycle of 3 of these cases. The authors of this study concluded that, although small, the majority of EFS was false and can be effectively recovered and lead to reasonable positive results (30). Further investigations have been conducted to put forth this issue; Griesinger et al. reported that EFS was seen in only one patient in a study of 51 cases after the freeze. In the same study, the live birth rate after the first freeze-thaw embryo transfer was reported as $5.9 \%$ in the slow freeze group and $19.4 \%$ in the vitrification group. In three cases, moderate OHSS (5.9\%) was diagnosed and an early onset severe OHSS developed in one case $(2 \%)$. The OHSS criterion was the Golan classification, and as a result of the study, the authors concluded that cryopreservation was an effective and safe method after triggering with agonist, although there was a severe early-onset OHSS case (31). These data showed us that empty follicle syndrome did not seem to be a drawback following the GnRHa trigger.

As we emphasized with this study, the GnRHa trigger has 
been considered to be an OHSS preventive measure. Several data have been presented on this issue; Fatemi et al. reported two severe cases of OHSS in the GnRH antagonist protocol in 2014 after the GnRH agonist without any hCG for luteal phase support. Based on these cases, GnRH, FSH and LH receptor mutations and the emergence of a clinical connection between the emergence and severity of OHSS and need to be aware that OHSS cannot be completely eliminated despite all the measures taken (32). In a retrospective study by Koren et al. in 2018, ovulation was triggered with the GnRH agonist instead of the classical hCG trigger in IVF patients with an E2 level of $\geq 3,000 \mathrm{pg} / \mathrm{mL}$. In this study, the authors applied the "Freeze-all" decision when the number of oocytes collected was 20 and above; on day 3 after oocyte collection, single bolus rescue hCG was administered for fresh embryo transfers. The authors found clinical pregnancy rate and OHSS rates similar in both groups at the end of the study and reported that both strategies seemed to be effective and safe, especially the upper limit of 20 oocytes was safe to implement a rescue hCG strategy (33). A study similar to ours was conducted in 2018 by Hwang et al. None of the 40 IVF patients who underwent GnRH analog trigger and the "Freeze-all" were reported to have moderate or severe ovarian hyperstimulation syndrome $(0 \%)$. At the end of the study, the authors reported the clinical pregnancy rate after frozen-thawed embryo transfer as $57.5 \%$ per cycle, and the cumulative rate of pregnancy after three embryos was $75.0 \%$ (34). In that study, the long-acting corifollitropin alpha was used for ovarian stimulation and followed by leuprolide acetate for ovulation trigger. Finally, Stormlund et al. are conducting a multicenter randomized, controlled, double-blind study with 424 normoresponder IVF patients aged 18 to 39 years from Denmark and Sweden. The results of fresh blastocyst (single) transfer will be compared with hCG triggers in gonadotropin-induced cyclic cases. The primary outcome of the study was reported to be as ongoing pregnancy rates and the results have not been reported yet (35).

The major disadvantage of this study was retrospective nature and small sample size. Cases were selected in a consecutive manner to overcome possible bias during patient selection. Further studies are needed to show live birth rates and newborn outcome following GnRH trigger.

In conclusion, the use of a protocol consisting of $\mathrm{GnRH}$ agonist trigger after $\mathrm{GnRH}$ antagonist cotreatment and the "Freeze-all" strategy reduces the risk of OHSS in high-risk patients undergoing IVF without affecting cycle characteristics and pregnancy rates.

Author contribution: GBN: Hypothesis, data collection, ET: Data collection, EO: Data collection, EC: Data collection, Manuscript preparation, FK: Data collection.

Acknowledgment: None. Conflict of interest: None Funding: None

\section{References}

1. Delvigne A, Rozenberg S. Review of clinical course and treatment of ovarian hyperstimulation syndrome (OHSS). Hum Reprod Update. 2003;9(1):77-96. doi: 10.1093/ humupd/dmg005.

2. Delvigne A, Rozenberg S. Epidemiology and prevention of ovarian hyperstimulation syndrome (OHSS): a review. Hum Reprod Update. 2002;8(6):559-77. doi: 10.1093/ humupd/8.6.559.

3. Wada I, Matson PL, Troup SA, Lieberman BA. Assisted conception using buserelin and human menopausal gonadotrophins in women with polycystic ovary syndrome. Br J Obstet Gynaecol. 1993;100(4):365-9. doi: 10.1111/j. 1471-0528.1993.tb12981.x.

4. Delvigne A, Demoulin A, Smitz J, Donnez J, Koninckx P, Dhont $\mathrm{M}$, et al. The ovarian hyperstimulation syndrome in in-vitro fertilization: a Belgian multicentric study. I. Clinical and biological features. Hum Reprod. 1993;8(9): 1353-60. doi: 10.1093/oxfordjournals.humrep.a138260.

5. Itskovitz-Eldor J, Kol S, Mannaerts B. Use of a single bolus of $\mathrm{GnRH}$ agonist triptorelin to trigger ovulation after GnRH antagonist ganirelix treatment in women undergoing ovarian stimulation for assisted reproduction, with special reference to the prevention of ovarian hyperstimulation syndrome: preliminary report: short communication. Hum Reprod. 2000;15(9):1965-8. doi: 10.1093/ humrep/15.9.1965.

6. De Jong D, Van Hooren EG, Macklon NS, Mannaerts BM, Fauser BC. Pregnancy and birth after GnRH agonist treatment for induction of final oocyte maturation in a woman undergoing ovarian stimulation for ICSI, using a GnRH antagonist (Orgalutran/Antagon) to prevent a premature LH surge: a case report. J Assist Reprod Genet. 2001;18(1):30-3. doi: 10.1023/a:1026498629324.

7. Kol S. Luteolysis induced by a gonadotropin-releasing hormone agonist is the key to prevention of ovarian hyperstimulation syndrome. Fertil Steril. 2004;81(1):1-5. doi: 10.1016/j.fertnstert.2003.05.032.

8. Rotterdam ESHRE/ASRM-Sponsored PCOS Consensus Workshop Group. Revised 2003 consensus on diagnostic criteria and long-term health risks related to polycystic ovary syndrome. Fertil Steril. 2004;81(1):19-25. doi: 10. 1016/j.fertnstert.2003.10.004.

9. Balen AH, Laven JS, Tan SL, Dewailly D. Ultrasound assessment of the polycystic ovary: international consensus definitions. Hum Reprod Update. 2003;9(6):505-14. doi: 10.1093/humupd/dmg044.

10. Cummins JM, Breen TM, Harrison KL, Shaw JM, Wilson LM, Hennessey JF. A formula for scoring human embryo growth rates in in vitro fertilization: its value in predicting pregnancy and in comparison with visual estimates of embryo quality. J In Vitro Fert Embryo Transf. 1986;3(5): 284-95. doi: 10.1007/BF01133388. 
11. Mackens S, Santos-Ribeiro S, van de Vijver A, Racca A, Van Landuyt L, Tournaye H, et al. Frozen embryo transfer: a review on the optimal endometrial preparation and timing. Hum Reprod. 2017;32(11):2234-42. doi: 10.1093/ humrep/dex 285 .

12. Golan A, Ron-el R, Herman A, Soffer Y, Weinraub Z, Caspi E. Ovarian hyperstimulation syndrome: an update review. Obstet Gynecol Surv. 1989;44(6):430-40. doi: 10. 1097/00006254-198906000-00004.

13. Babayof R, Margalioth EJ, Huleihel M, Amash A, ZylberHaran E, Gal M, et al. Serum inhibin A, VEGF and TNFalpha levels after triggering oocyte maturation with GnRH agonist compared with HCG in women with polycystic ovaries undergoing IVF treatment: a prospective randomized trial. Hum Reprod. 2006;21(5):1260-5. doi: 10.1093/humrep/dei475.

14. Fauser BC, de Jong D, Olivennes F, Wramsby H, Tay C, Itskovitz-Eldor $\mathrm{J}$, et al. Endocrine profiles after triggering of final oocyte maturation with GnRH agonist after cotreatment with the GnRH antagonist ganirelix during ovarian hyperstimulation for in vitro fertilization. J Clin Endocrinol Metab. 2002;87(2):709-15. doi: 10.1210/jcem. 87.2.8197.

15. Beckers NG, Macklon NS, Eijkemans MJ, Ludwig M, Felberbaum RE, Diedrich K, et al. Nonsupplemented luteal phase characteristics after the administration of recombinant human chorionic gonadotropin, recombinant luteinizing hormone, or gonadotropin-releasing hormone (GnRH) agonist to induce final oocyte maturation in in vitro fertilization patients after ovarian stimulation with recombinant follicle-stimulating hormone and GnRH antagonist cotreatment. J Clin Endocrinol Metab. 2003;88 (9):4186-92. doi: 10.1210/jc.2002-021953.

16. Lanzone A, Fulghesu AM, Apa R, Caruso A, Mancuso S. LH surge induction by GnRH agonist at the time of ovulation. Gynecol Endocrinol. 1989;3(3):213-20. doi: 10. $3109 / 09513598909152302$.

17. Humaidan P, Bredkjaer HE, Bungum L, Grondahl ML, Westergaard L, Andersen CY. GnRH agonist (buserelin) or hCG for ovulation induction in GnRH antagonist IVF/ ICSI cycles: a prospective randomized study. Hum Reprod. 2005;20(5):1213-20. doi: 10.1093/humrep/deh765.

18. Kolibianakis EM, Schultze-Mosgau A, Schroer A, van Steirteghem A, Devroey P, Diedrich K, et al. A lower ongoing pregnancy rate can be expected when GnRH agonist is used for triggering final oocyte maturation instead of HCG in patients undergoing IVF with GnRH antagonists. Hum Reprod. 2005;20(10):2887-92. doi: 10.1093/humrep/dei150.

19. Griesinger G, Diedrich K, Devroey P, Kolibianakis EM. $\mathrm{GnRH}$ agonist for triggering final oocyte maturation in the GnRH antagonist ovarian hyperstimulation protocol: a systematic review and meta-analysis. Hum Reprod Update. 2006;12(2):159-68. doi:10.1093/humupd/dmi045.

20. Le MT, Nguyen DN, Zolton J, Nguyen VQH, Truong QV,
Cao NT, et al. GnRH Agonist versus hCG Trigger in Ovulation Induction with Intrauterine Insemination: A Randomized Controlled Trial. Int J Endocrinol. 2019 Mar 13;2019:2487067. Doi: 10.1155/2019/2487067.eCollecti on 2019.

21. Zhu Q, Chen Q, Wang L, Lu X, Lyu Q, Wang Y, et al. Live birth rates in the first complete IVF cycle among 20 687 women using a freeze-all strategy. Hum Reprod. 2018;33(5):924-9. doi: 10.1093/humrep/dey044.

22. Ferraretti AP, Gianaroli L, Magli C, Fortini D, Selman HA, Feliciani E. Elective cryopreservation of all pronucleate embryos in women at risk of ovarian hyperstimulation syndrome: efficiency and safety. Hum Reprod. 1999;14 (6):1457-60. doi: 10.1093/humrep/14.6.1457.

23. Roque M, Lattes K, Serra S, Solà I, Geber S, Carreras R, et al. Fresh embryo transfer versus frozen embryo transfer in vitro fertilization cycles: a systematic review and metaanalysis. Fertil Steril. 2013;99(1):156-62. doi: 10.1016/j. fertnstert.2012.09.003.

24. Shi Y, Sun Y, Hao C, Zhang H, Wei D, Zhang Y, et al. Transfer of Fresh versus Frozen embryos in Ovulatory Women. N Engl J Med. 2018;378(2):126-36. doi: 10.10 56/NEJMoa1705334.

25. Vuong LN, Dang VQ, Ho TM, Huynh BG, Ha T, Pham TD, et al. IVF transfer of fresh or frozen embryos in women without polycystic ovaries. N Engl J Med. 2018; 378(2):137-47. doi: 10.1056/NEJMoa1703768.

26. Chen ZJ, Shi Y, Sun Y, Zhang B, Liang X, Cao Y, et al. Fresh versus frozen embryos for infertility in the polycystic ovary syndrome. N Engl J Med. 2016;375(6):523-33. doi: 10.1056/NEJMoa1513873.

27. Tomás C, Alsbjerg B, Martikainen H, Humaidan $\mathrm{P}$. Pregnancy loss after frozen-embryo transfer-a comparison of three protocols. Fertil Steril. 2012;98(5):1165-9. doi: 10.1016/j.fertnstert.2012.07.1058.

28. Wennerholm UB, Henningsen AK, Romundstad LB, Bergh C, Pinborg A, Skjaerven R, et al. Perinatal outcomes of children born after frozen-thawed embryo transfer: a Nordic cohort study from the CoNARTaS group. Hum Reprod. 2013;28(9):2545-53. doi: 10.1093/humrep/ $\operatorname{det} 272$.

29. Krishna D, Dhoble S, Praneesh G, Rathore S, Upadhaya A, Rao K. Gonadotropin-releasing hormone agonist trigger is a better alternative than human chorionic gonadotropin in PCOS undergoing IVF cycles for an OHSS Free clinic: a randomized control trial. J Hum Reprod Sci. 2016;9(3):164-72. doi: 10.4103/0974-1208.192056.

30. Deepika K, Sindhuma D, Kiran B, Ravishankar N, Gautham P, Kamini R. Empty Follicle Syndrome Following GnRHa Trigger in PCOS Patients Undergoing IVF Cycles. J Reprod Infertil. 2018;19(1):16-25. PMID: 29850443. PMID: 29850443.

31. Griesinger G, Schultz L, Bauer T, Broessner A, Frambach $\mathrm{T}$, Kissler S. Ovarian hyperstimulation syndrome preven- 
tion by gonadotropin-releasing hormone agonist triggering of final oocyte maturation in a gonadotropin-releasing hormone antagonist protocol in combination with a "freeze-all" strategy: a prospective multicentric study. Fertil Steril. 2011;95(6):2029-33. doi: 10.1016/j.fertnstert. 2011.01.163.

32. Fatemi HM, Popovic-Todorovic B, Humaidan P, Kol S, Banker M, Devroey P. Severe ovarian hyperstimulation syndrome after gonadotropin-releasing hormone $(\mathrm{GnRH})$ agonist trigger and "freeze-all" approach in GnRH antagonist protocol. Fertil Steril. 2014;101(4):1008-11. doi: 10. 1016/j.fertnstert.2014.01.019.

33. Rahav Koren R, Gonen O, Hershko Klement A, Haikin Herzberger E, Ghetler Y, Shulman A, et al. Number of oocytes retrieved as a criterion for "freeze-all" strategy versus a single "rescue" bolus of low-dose human chori- onic gonadotropin following GnRH agonist for ovulation triggering: a pilot study. Gynecol Obstet Invest. 2018; 83(5):471-76. doi: 10.1159/000479557.

34. Hwang JL, Chen SU, Chen HJ, Chen HF, Yang YS, Chang $\mathrm{CH}$, et al. Feasibility of corifollitropin alfa/GnRH antagonist protocol combined with GnRH agonist triggering and freeze-all strategy in polycystic ovary syndrome patients. J Formos Med Assoc. 2018;117(6):535-40. doi: 10.1016/ j.jfma.2017.05.009.

35. Stormlund S, Løssl K, Zedeler A, Bogstad J, Prætorius L, Nielsen HS, et al. Comparison of a 'freeze-all' strategy including GnRH agonist trigger versus a 'fresh transfer' strategy including hCG trigger in assisted reproductive technology (ART): a study protocol for a randomised controlled trial. BMJ Open. 2017;7(7):e016106. doi: 10. 1136/bmjopen-2017-016106. 